\title{
Discourse Analysis of a Moodle Based Academic Discussion Among Student Community
}

\author{
Virendra Singh Nirban, Birla Institute of Technology and Science, Pilani, India \\ Priyanka Kumari, Birla Institute of Technology and Science, Pilani, India
}

\begin{abstract}
The last two decades have witnessed an unprecedented growth in the number of users going online. While it has not happened overnight, the growth has been exponential in the last one decade. Chronologically, New media revolution started with the advent of internet. Innovations in computer and network technologies led to a massive adoption of communication technologies in varied forms. The scene in education domain is no different. There is lot of interest and investment in technology integrated education. With innovations such as MOODLE and MOOCS, the opportunities have grown manyfold. New media has enabled the end user to make a transition from a mere information consumer to a spontaneous information producer. Moreover, all along, new media has broken the space and time barrier leading to ubiquitous environment to communicate. It is in this context that the discourse generated through the new media is becoming an object of interest from research perspective. This paper uses a MOODLE based university Learning Management System and its discussion forums to make students participate in online discussions. The resulting academic discourse has been analysed using content analysis through a facet based approach. The objective of the study is to understand how technology and social aspects of an academic discussion are manifested in a mediated discourse.
\end{abstract}

Keywords: Computer-mediated Communication, New Media, Online Discourse, Content Analysis, Learning Management System, Discussion Forum 


\section{Introduction}

The last two decades have witnessed an unprecedented growth in the number of users going online. While it has not happened overnight, the growth has been exponential in the last one decade. Chronologically, New media revolution started with the advent of internet. Innovations in computer and network technologies led to a massive adoption of communication technologies in varied forms. The scene in education domain is no different.

Technology intervention in education is not a new concept. Technology has been used in various forms and for various purposes to make teaching and learning more effective. Starting from radio and television to audio recordings and Video cassette players, technology has been used in classrooms for various purposes. This is particularly true for language and communications disciplines. With the advent of computers and internet as a mainstream technologies, new and varied platforms of communication and collaboration are available to teachers and students. The teachers and learners can not only consume content but also create content of their own which can be shared and disseminated.

There is lot of interest and investment in technology integrated education. With innovations such as MOODLE and MOOCS, the opportunities have grown manyfold. New media has enabled the end user to make a transition from a mere information consumer to a spontaneous information producer. Moreover, all along, new media has broken the space and time barrier leading to ubiquitous environment to communicate. It is in this context that the discourse generated through the new media is becoming an object of interest from research perspective.

This paper uses a MOODLE based university Learning Management System and its discussion forums to make students participate in online discussions. The resulting academic discourse has been analysed using content analysis through a facet based approach. The objective of the study is to understand how technology and social aspects of an academic discussion are manifested in a mediated discourse.

\section{Literature Review}

Research in the area of New Media enabled education suggests that there are numerous opportunities that remains unutilized in higher education. Level and Hoseth (2008) argue that about 90 percent of the college students in the U.S. have gone online and thus are the 
members of the so called 'net-generation' who are surrounded with technology and internet so much so that these have affected almost every facet of their lives. Obviously not all are technology savvy individuals but majority of the students, given their familiarity with technology and the internet, are 'digital natives' as opposed to 'digital immigrants' (Kvavik, 2005; as quoted by Level \&Hoseth, 2008).Further discussing in the same context, Richardson (2007) argues that learning with the help of Web 2.0 is the process of building connections where sharing, filtering and saving the relevant information can help build one's own curriculum. Olarinan (2008) argues that Web 2.0 facilitates a high cognitive thinking as its 'browse' feature enables students to learn while they seek information.

The curiosity that Web 2.0 arouses leads to enhanced knowledge acquisition. Web 2.0 facilitates social constructivist pedagogy (Rosen \& Nelson, 2008;Huang et al., 2009; Newland \&Byles, 2013) and creates a 'prod-user' identity where users are also the producers of learning resources (Brown, 2008;Huang et al., 2009). Hence, unlike the previous version of web, Web 2.0 invites participation and enables users to contribute content, thereby, giving equal opportunity to every user. Another major opportunity that Web 2.0 offers is that it empowers individuals by facilitating learner-controlled learning environment (Brown, 2008; Crummett\& Perrault, 2008) where students learn through interaction (Rosen \& Nelson, 2008; Su \& Garcia, 2008; Huang et al., 2009; Churchill, 2011), collaboration(Richardson, 2007; Rosen \& Nelson, 2008; Su \& Garcia, 2008; Huang et al., 2009; Churchill, 2011; Kale, 2013), and sharing (Richardson, 2007; Rosen \& Nelson, 2008; Churchill, 2011). Kit Hang Leung (2008), in his research, finds out that high order thinking emerges in online discussions, because when the discussants discuss ideas, they ponder over and expand the existing concepts and in this process, new ideas and concepts emerge. Further user participation increases owing to the ease of use and social networking options of Web 2.0 (Rosen\& Nelson, 2008; Su \& Garcia, 2008). Web 2.0 offers the opportunity to interact beyond time/space barriers (Crummett\& Perrault, 2008; Price, 2008;Risquez, 2008), and this flexibility, in turn, offers extended opportunities of participation (Risquez, 2008). Crummett and Perrault (2008) even argue that since most of Web 2.0 tools and applications are available free, they provide more opportunities of participation and interaction.For implementing Web 2.0 in higher education, Olarinan (2008) argues for blending CMC with traditional face-to-face interactions, for it would give students time to adapt to the CMC system. 
In the social context, the interactive features of Web 2.0 lead to strengthened bonding between students and teachers (Churchill, 2011). While collaboration in the educational context facilitates learning by building 'collective wisdom' (Rosen \& Nelson, 2008) and sharing of 'expertise' and resources (Risquez, 2008); in the social context, it enables the emergence of a feeling of community or belonging which is extremely advantageous for efficient learning (Su \& Garcia, 2008).Education through Web 2.0 promotes self-expression, increases motivation and inquiry skills of students (Kale, 2013) and provides opportunity to those students also who are either shy or uncomfortable in expressing themselves in the faceto-face classroom (Price, 2008).

\section{Methodology}

A MOODLE based Learning Management System(LMS) of a private university was used as a new media platform for this study. The LMS offered the utility of online discussions. An elective course, named Computer-Mediated Communication, was offered to the students. 85 students registered in the course. The students were asked to participate in an online discussion. Though the discussion was an evaluation component in the course, it was offered as an out-of the class activity. 81 students participated in the discussion. The discussion was initiated by the instructor. Students were given 48 hours to participate in the discussion. At the end of the discussion data was compiled and statistical as well as qualitative analysis was carried out.

\section{Findings and Discussion}

At the end of the discussion data was compiled and statistical as well as qualitative analysis was carried out. The discussion threads were identified; numbers of posts per user were counted. Of the total posts per user, the reactions/feedback and threads initiated by the user were counted separately. Apart from quantitative contribution, the substance of the contribution was also observed during the analysis.

To begin with, the topic of the discussion was:

"CMC has been widely accepted as an enabling force in the context of communication across the globe. CMC is providing a powerful platform to users to participate and influence all aspects of life such as information 
creation and consumption, politics, religion, freedom, entertainment, culture etc. But at the same time, there are dysfunctions also."

It was expected from the students that they comprehend the breadth of the topic and pick appropriates aspects for discussions. They should also be able to identify the dysfunction contrast in the statement. The number of total posts by a student, initiations by a students and replies to others participants were counted for the entire course. Fig 1 shows the frequency of posts by students. The figure shows that approximately $26 \%$ of the students posted only once, $17 \%$ posted twice, $22 \%$ posted between three to five times, $26 \%$ posted between six to 12 times. The highest number of posts was 32 by one student. The data implies that the participation ws skewed and was not uniform. A majority of student didn't participate beyond couple of times. The concept of discussion and its dynamics were discussed in the classroom and the students were given 48 hours of duration to contribute. Although there were a total of 418 posts contributed by the entire course team but the distribution of discussion was not uniform.

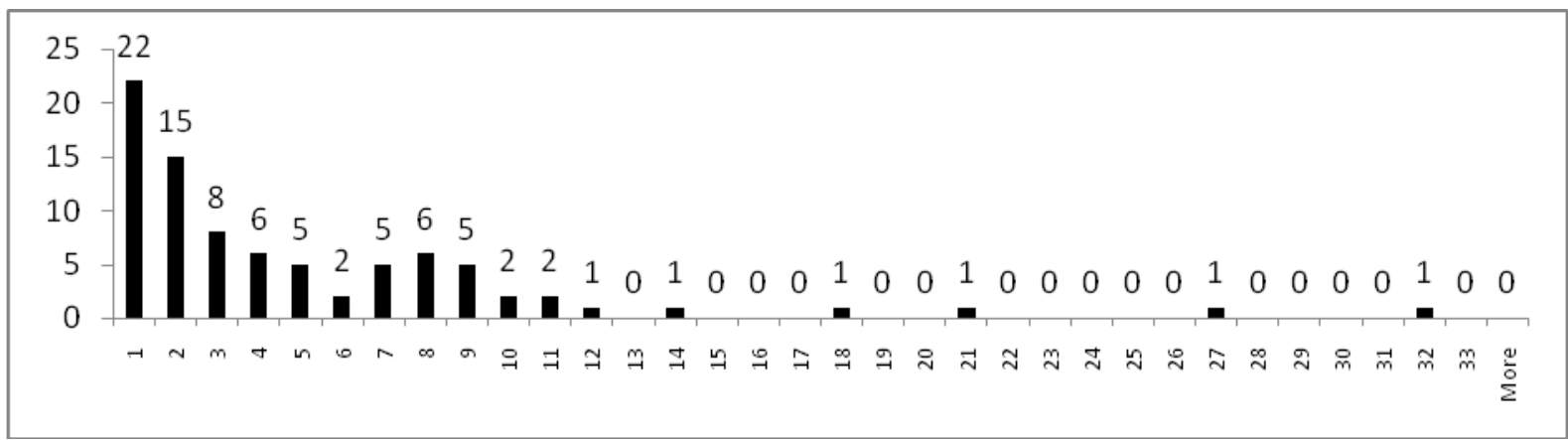

Figure 1. Frequency of posts in the discussion

Turn taking is an important aspect of conversation communication. Since discussion is also a type of conversation, albeit argumentative in nature, turn taking becomes important. Turn taking in an online discussion may be measured through he count of frequency of replies. Figure 2 shows the reply frequency of the participants. The graph shows that an overwhelmingly $71 \%$ of the students replied only once and the remaining $29 \%$ were distributed in the reply frequency of two to 12. This data implies that the students really didn't get into the nuances of the topic. It also implies that a minority of the participants were discussing the topic among themselves and that the remaining once were isolated from them. 
Consequently, with this kind of behaviour the impact of technology in learning would be minimal.

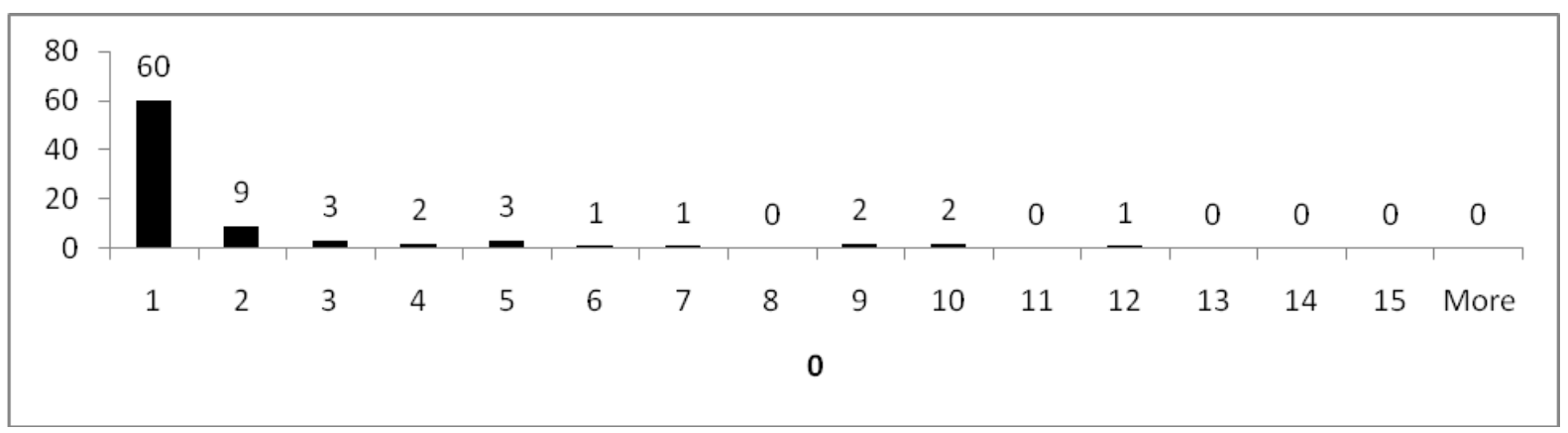

Figure 1. Frequency of replies in the discussion

Apart from turn taking, raising an argument and starting a topic thread which helps in covering the breadth of the topic, is also an important aspect of discussion skills. This can be measured in an online discussion from the counted frequencies of new threads started from the original one. Figure 3 shows the initiation frequency of the participants in the context of the topic. It can be deduced from the figure that $53 \%$ of the participants initiated only once or twice, $34 \%$ initiated between three to seven times and the remaining were distributed between eight to 22 times. This again reflects that un-interactive behaviour of the students in the group. A majority of the students were concerned about contributing a 'post' rather than an argument or an explanation to an argument by self or other participants.

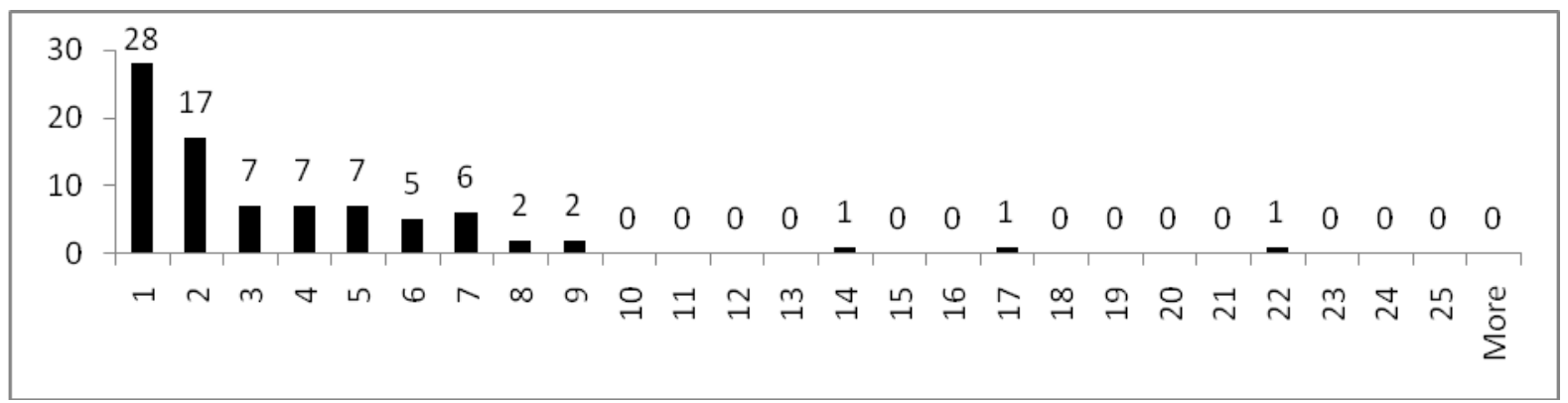

Figure 1. Frequency of initiations in the discussion

A closer look at the data shows that overall there were 418 posts made by 73 students. This turns out to be around 5.7 posts per students which is impressive. But this average statistical participation is undone by the individual contribution of the participants. The participation very skewed. Similarly average initiations stand at 3.9 which again is very impressive and 
suggestive of substantial participation. In this case again the students were not as forthcoming as the data suggests. All this implies that the dynamics of a discussion were not reflected in the discussion activity carried out via technology intervention. It seems a small population of the classroom was more active than the rest of the class population. It also implies that the technology intervention may bear better results if smaller groups are engaged.

The nature of participation among students can also be understood from New Media Theories also. Ulnan and Markus (1987) coined the term Cues-Filtered-Out Theories to describe a set of theories which explain communication interaction via new media technologies. All these theories propagates the view that computer technologies have varied capabilities to transmit nonverbal cues, i.e. technologies vary in their message carrying capacities. It implies that certain nonverbal cue systems are not transmitted and therefore certain social functions can't be achieved to the desired effect. In an activity such as discussion, the intensity of argument is also affected by the non-verbal and para-verbal aspects of communication. A technology like text based discussion forum severely limits transmission of nonverbal cues. Consequently, participants may not feel as engaged and involved in the task.

Herring (2007) proposed the faceted approach to understand and classify the mediated discourse. She proposed two main dimensions, namely - technology and social., with several facets for each of the dimensions to understand the nuances of the generated discourse. Table 1 provides summarised presentation of the characterization of the discussion on several facets proposed by Herring.

Table 1. Discourse characterization using Herring's Faceted Approach

\begin{tabular}{|c|c|c|c|}
\hline \multicolumn{4}{|c|}{ Technology Dimension } \\
\hline Facet & Value & Facet & Value \\
\hline Synchronicity & Not Supported & $\begin{array}{c}\text { Channels of } \\
\text { Communication }\end{array}$ & Text Only \\
\hline Message & Multidirectional / & Anonymity & Not Supported \\
Transmission & Broadcast & & Heap \\
\hline Persistence & Permanent & Message Format & \\
\hline Message Size & Unlimited & & \\
\hline
\end{tabular}




\begin{tabular}{|c|c|c|c|}
\hline \multicolumn{4}{|c|}{ Social Dimension } \\
\hline Facet & Value & Facet & Value \\
\hline $\begin{array}{c}\text { Participant } \\
\text { structure }\end{array}$ & $\begin{array}{c}\text { Large group, public } \\
\text { part. }\end{array}$ & Tone & Formal \\
\hline $\begin{array}{c}\text { Participant } \\
\text { characteristics }\end{array}$ & Same age group & Activity & Discussion \\
\hline Purpose & Social, Academic & Norms & Original contribution, formal \\
& & & language \\
\hline Theme & Topical & Code & English \\
\hline
\end{tabular}

From Table 1, it can be opined that the activity (discussion) was carried out in an asynchronous mediated environment which provided response time flexibility to the participants and therefore the expected substantial contribution by the active participants in terms of argumentative and expository discourse. The message transmission happened in a broadcast fashion which implies that it was a publicly generated discourse. This made participants more context aware. The size of the posts was unlimited and therefore many instances of lengthy arguments in the form of long paragraphs were observed in the discussion. This is equivalent to an undesirable lengthy monologue in an oral discussion. Anonymity was not allowed which reduced bogus and deviated arguments in the discussion. The format of the discussion was 'last in at the top of heap' which presented the discourse in sequential and chronological order. This also allowed to assess the duration between the posts by individual participants.

With respect to social dimension, the participants belonged to same age group and the participant structure consisted of a large, academically heterogenous group. This resulted in a varied level of intensity in the arguments and message composition. It may also have affected the skewed distribution of the contribution in the discussion. Since the theme of the discussion was topical, there were minima digressions and the discourse that was generated was also academic in nature. Since the activity was part of the course, the formal code of discussion was English and the participants also adhered to formal use of English. The course norms, in terms of original contribution and formal discussion, were also observed among the participants. 
It can be inferred from the faceted explanation of the discussion discourse that apart from participant skills and the dynamics of the activity, the technology properties and social characteristics of the participating group may also influence the outcome of the activity and the kind of discourse that is generated.

\section{Conclusions}

This study used a MOODLE based LMS to conduct an online discussion among the students registered in a course. It may be concluded from the short study that the intervention of technology in education in general and in language and communication discipline in particular is still a work of experiment. The technology use is still evolving. In this particular case, although the average participation seems okay but overall participation and discussion distribution is poor. Also the constructive nature of discussion activity and the consensus building exercise was not reflected among the participants. These observations are very specific to this study and can not be generalised. More studies using smaller and varied groups are required to make a more generalised conclusion about new media technology usage in inculcating written communication skills. 


\section{References}

Brown, Stephen."From VLEs to Learning Webs: The Implication of Web 2.0 for Learning and teaching."Interactive Learning Environments18.1(2010): 110.Taylor\&Francis.Web. 9 Jul.2008.

Churchill, Daniel."Web2.0 in Education: A Study of the Explorative Use of Blogs with a Postgraduate Class."Innovations inEducation andTeaching International 48.2 (2011): 149-158. Taylor \&Francis. Web.18 May2011.

Crummett, Courtney D., Anna H.Perrault.“The Useof CMC Technologies in Academic Libraries."Handbook ofResearch on Computer Mediated Communication.Sigrid Kelsey\&Kirk St. Amant.Vol2, 2008.705-719.

Culnan, M. J., \& Markus, M. L. (1987). Information technologies. In F. M. Jablin, L. L. Putnam, K. H. Roberts, \& L. W. Porter (Eds.), Handbook of organizational communication: An interdisciplinary perspective (pp. 420-443). Newbury Park, CA: Sage.

Huang, Yueh-Min, Stephen J. H. Yang, andChin-Chung Tsai."Web 2.0 for Interactive Learning."Interactive LearningEnvironments17.4(2009): $\quad$ 257-259.Taylor\& Francis.Web.10 Nov. 2009.

Kale, Ugur. "Can TheyPlan to Teach With Web 2.0?Future Teachers' Potential Use of theEmerging Web."Technology, Pedagogy and Education (2013): 1-19.Taylor \& Francis.Web.14 Aug. 2013.

Level, Allison V.,Amy E. Hoseth."Learning and Teaching with CMC in the U.S.Higher Education Arena."Handbook of Research on Computer Mediated Communication.SigridKelsey\&Kirk St. Amant.Vol1, 2008. 34-48.

Newland, Barbara; Byles, Linda. "Changing AcademicTeaching with Web 2.0 Technologies."Innovations in Education and Teaching International (2013).Taylor\&Francis.Web.14 May 2013.

Olarinan, BolanleA."Human Computer Interaction \& the Best Mixes of Face-to-Face and EInteractions in Educational Settings." Handbook of Research on Computer Mediated Communication.Sigrid Kelsey \& Kirk St. Amant.Vol1, 2008. 49-61.

Olarinan, Bolanle A. "Culture, LearningStyles and Web 2.0." Interactive Learning Environments 17.4(2009): 261-271.Taylor \& Francis. Web.10 Nov. 2009. 
Price, Apryl C. "Higher Education's Use of Course Management Software.” Handbook of Research on Computer Mediated Communication. Sigrid Kelsey \& Kirk St. Amant.Vol 1, 2008.62-72.

Richardson, Will.“Teaching in a Web 2.0World."Kappa Delta Pi Record 43.4(2007): 150151.Taylor\& Francis.Web 13Jul. 2012.

Risques, Angelica (2008)."E-Mentoring: An Extended Practice, an Emerging Discipline."Handbook of Research on Computer Mediated Communication. Sigrid Kelsey \& Kirk St. Amant.Vol2, 2008. 656-677.

$\mathrm{Su}$, Cheng-Chao, Karen Garcia."Chat Roomsfor Language Teaching and Learning."Handbook of Research on Computer Mediated Communication.Sigrid Kelsey\&Kirk St. Amant.Vol2, 2008.947- 968.

Herring, S. "A Faceted Classification Scheme for Computer-Mediated Discourse". Language@Internet, 2007. 